\title{
Produção de mudas de alfazema-do-Brasil (Aloysia gratissima) por estaquia sob aplicação de ácido indolbutírico (AIB)
}

O uso da estaquia é comum na propagação de plantas, principalmente por eliminar as desvantagens da propagação sexuada. O fator essencial para o sucesso na propagação por estaquia está na capacidade de enraizamento das estacas. Deste modo, é comum a aplicação exógena de fitorreguladores na estaquia, tal como o ácido indolbutírico (AlB), com o objetivo estimular um melhor enraizamento das estacas. Este trabalho teve como objetivo avaliar o efeito de três concentrações de ácido indolbultírico na formação e desenvolvimento do sistema radicular e brotações foliares em diferentes classes de estacas caulinares de alfazema-do-brasil (Aloysia gratissima). Os ramos de alfazema foram coletados e separados em estacas herbáceas, semilenhosas e lenhosas. As estacas foram desinfestadas em solução de hipoclorito de sódio $1 \%$ e submetidas aos tratamentos com AIB nas concentrações de 0,1500 e 3000 ppm. Utilizou-se esquema fatorial $3 \times 3$ (concentraç̃o $\times$ tipo de estaca) com três blocos, sendo o delineamento adotado no experimento em blocos casualizados. Foram avaliadas as taxas de sobrevivência das estacas, de enraizamento e de brotações foliares, além do número médio de raízes e o comprimento médio da maior raiz. As estacas herbáceas não sobreviveram ao período de aclimatação. Já as semi-lenhosas e lenhosas tiveram baixa taxa de sobrevivência e mostraram-se pouco eficientes no enraizamento sob efeito do AlB. Os melhores resultados foram encontrados na concentração de 0 ppm, principalmente em estacas semi-lenhosas, com maior ocorrência de estacas vivas, enraizadas e com brotações. As taxas de eficiência observadas nos parâmetros analisados podem estar associadas ao tempo de enraizamento, condições ambientais e às doses de AIB aplicadas, uma vez que as concentrações utilizadas podem ter resultado em efeito inibitório.

\section{Production of alfazema-do-Brasil seedlings (Aloysia gratissima) by staquia under indolbutyric acid (AIB) application}

\begin{abstract}
The use of cuttings is common in plant propagation, mainly because it eliminates the disadvantages of sexual propagation. The essential factor for successful propagation by cuttings is the rooting capacity of the stakes. In this way, is common an exogenous application of phytoregulators in the cuttings, such as indolbutyric acid (IBA), in order to stimulate a better rooting of the cuttings. This work aimed to evaluate the effect of three concentrations of indolbultiric acid on the formation and development of the root system and leaf sprouts in different classes of in a $1 \%$ sodium hypoch orite solution and subjected to treatments with IBA a concentrations of 0,1500 and $3000 \mathrm{ppm}$. A 3x3 factorial scheme (concentration $x$ type of pile) with three blocks was used, with the design adopted in the experiment in randomized blocks. Cuttings survival rates, rooting and leaf buds were evaluated, as well as the average number of roots and the average length of the largest root. The herbaceous cuttings did not survive to the acclimatization period. The semi-hardwoods and woody ones had a low survival rate and proved to be inefficient in rooting under the effect of AIB. The best results were found in the concentration of 0 ppm, mainly in semi-hardwood cuttings, with a higher occurrence of live, rooted cuttings with sprouts. . The efficiency rates observed in the analyzed parameters may be associated with the rooting time, environmental conditions and the applied IBA doses, since the concentrations used may have resulted in an inhibitory effect.
\end{abstract}

Keywords: Alfazema; Propagation; Stake; Phytoregulator.

Mayhara da Costa Pereira (iD

Universidade Federal Rural do Rio de Janeiro, Brasil http://lattes.cnpq.br/2943346567254211 http://orcid.org/0000-0003-1595-1194 mayhara-pereira@hotmail.com

Lucas de Souza da Silva (iD)

Universidade Federal Rural do Rio de Janeiro, Brasil http://lattes.cnpq.br/3760372132788863 http://orcid.org/0000-0003-2117-9794

desouza.lucas2018@gmail.com

\section{Débora Fernandes da Graça Mello}

Universidade Federal Rural do Rio de Janeiro, Brasil http://lattes.cnpq.br/1460127973103240 deboragmello@gmail.com
Tadeu Augusto van Tol de Castro (iD

Universidade Federal Rural do Rio de Janeiro, Brasil http://lattes.cnpq.br/6353947754324048 http://orcid.org/0000-0003-4095-3976 tadeuvantol@hotmail.com

Rafael Gomes da Mota Gonçalves (iD

Universidade Federal Rural do Rio de Janeiro, Brasil http://lattes.cnpq.br/0244672026134100 http://orcid.org/0000-0001-9541-6698

rafaelmotag@hotmail.com

Hellen Fernanda Oliveira da Silva

Universidade Federal Rural do Rio de Janeiro, Brasil http://lattes.cnpq.br/8215713473257692

http://orcid.org/0000-0003-4618-3984

hellenfosagro@gmail.com
Danielle França da Oliveira (iD)

Universidade Federal Rural do Rio de Janeiro, Brasil http://lattes.cnpq.br/5563595706020944 http://orcid.org/0000-0002-5336-4241 dani.foliveira@hotmail.com

Carlos Alberto Bucher

Universidade Federal Rural do Rio de Janeiro, Brasil http://lattes.cnpq.br/9011597325479363 http://orcid.org/0000-0002-2746-816X

carlos.bucher@gmail.com
Referencing this:

PEREIRA, M. C.; SILVA, L. S.; MELLO, D. F. G.; CASTRO, T. A. V. T.; GONCCALVES, R. G. M.; SILVA, H. F. O.; OLIVEIRA, D. F.; BUCHER, C. A. Produção de mudas de alfazema-do-Brasil (Aloysia gratissima) por estaquia sob aplicação de ácido indolbutírico (AIB). Nature and Conservation, v.14, n.1, p.116-122, 2021. DOI: http://doi.org/10.6008/CBPC2318-2881.2021.001.0013 


\section{INTRODUÇÃO}

Nas últimas décadas, foi observado um grande interesse pelo potencial terapêutico das plantas medicinais (YUNES et al., 2001). Tal fato é comprovado pela evidência que cerca de $30 \%$ das drogas prescritas no mundo são obtidas a partir de plantas. Além disso, cerca 50\% das drogas desenvolvidas entre 1981 e 2002 foram obtidas de produtos naturais ou análogos semissintéticos (KOEHN et al., 2005).

Tem-se verificado um grande avanço científico envolvendo estudos químicos e farmacológicos de plantas medicinais, visando a obtenção de novos compostos com propriedades farmacêuticas (CECHINEL FILHO et al., 1998). Dentre estes compostos, se destacam os constituintes químicos existentes nos óleos essenciais das plantas, pois pertencem ao maior e mais diversificado grupo dentro dos produtos naturais, apresentando uma grande importância terapêutica e econômica (SILVA et al., 2003).

A Aloysia gratissima é uma espécie que vem sendo cada vez mais estudada por possuir propriedades medicinais. É nativa da América do Sul, sendo encontrada também em diferentes regiões da América do Norte. Pertence à família Verbenaceae, sendo mais conhecida como "alfazema-do-brasil" (CARDOSO, 2005). É comumente utilizada na medicina popular, por apresentar propriedades diaforéticas, digestivas, tônicas, antigripais, calmante e antimicrobianas. Além disso, é uma espécie melífera e ornamental, devido à intensidade da floração e ao aroma agradável das flores (RICCIARDI et al., 2006). Contudo, é ainda pouco estudada em relação aos hábitos de crescimento e desenvolvimento, com literatura principalmente associada ao perfil químico da planta.

Para estimular o enraizamento e viabilizar a produção de mudas por meio de estaquia, é comum a utilização de reguladores de crescimento (FACHINELLO et al., 1995). É necessário um balanço hormonal adequado para que ocorra o processo de iniciação radicular, devendo haver equilíbrio entre inibidores e promotores de crescimento, principalmente entre auxinas, citocininas e giberelinas (PASQUAL, 2001; TAIZ et al., 2017).

Um dos meios mais utilizados para adequar o balanço hormonal visando o enraizamento se dá pela aplicação exógena de fitorreguladores, tais como o ácido indolbutírico (AIB). O AIB corresponde a uma auxina sintética, de maior estabilidade e menor solubilidade que a auxina endógena (ácido indolacético), sendo considerado um dos melhores estimuladores de enraizamento (PASQUAL et al., 2001; FERRIANI et al., 2006; PEREIRA et al., 2012).

Neste contexto, este trabalho teve o objetivo de avaliar o efeito de três doses de ácido indolbutírico (0, 1500 e 3000 ppm) na formação e desenvolvimento do sistema radicular e brotações foliares em diferentes classes de estacas caulinares (herbáceas, semi-lenhosas e lenhosas) de alfazema-do-brasil (Aloysia gratissima).

\section{MATERIAIS E MÉTODOS}

O experimento foi realizado no setor de Horticultura da Universidade Federal Rural do Rio de Janeiro

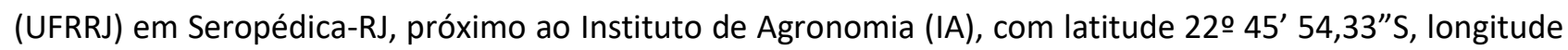


43은 41'53"O, e altitude de $33 \mathrm{~m}$ (Figura 1A), sendo o clima classificado como do tipo Aw na classificação de Köppen. Os ramos de alfazema-do-brasil (Aloysia gratissima) foram obtidos a partir de plantas matrizes, separados e categorizados de acordo com o grau de lignificação, em herbáceo, semi-lenhoso e lenhoso.

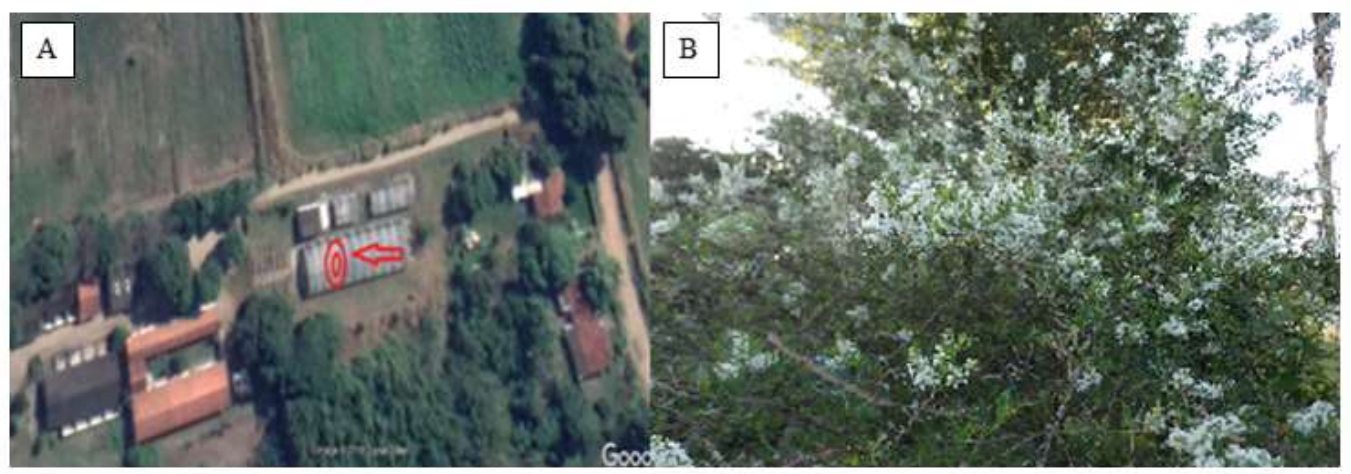

Figura 1: A) Localização do experimento; B) Planta matriz de Aloysia gratissima.

As três categorias de estacas obtidas foram padronizadas com $20 \mathrm{~cm}$ de comprimento e cortadas em bisel na extremidade basal. As estacas foram desinfestadas, sendo imergidas em solução de hipoclorito de sódio $1 \%$ por um minuto. As soluções de AIB (0, 1500 e 3000ppm) foram preparadas a partir da dissolução de ácido indolbutírico em etanol 50\%. Posteriormente, as extremidades inferiores das estacas foram submetidas ao contato com as soluções de ácido indolbutírico (AIB) nas concentrações de 0, 1500 e 3000 ppm, durante quinze segundos.

As estacas foram levadas ao propagador, sendo posicionadas a cerca de 1/4 de seu comprimento, utilizando areia lavada como substrato. A irrigação se deu por nebulização intermitente, mantendo a umidade constante, permanecendo nessas condições por aproximadamente 50 dias. Posteriormente, foram avaliadas a taxa de sobrevivência das estacas, a taxa de estacas enraizadas, a taxa de estacas com brotações, o número médio de raízes e o comprimento médio da maior raiz.

O experimento foi conduzido em blocos casualizados em esquema fatorial $3 \times 3$, sendo três concentrações (0, 1500 e 3000 ppm) e três tipos de estacas (herbáceo, semi-lenhoso e lenhoso), com três blocos. Para cada tratamento (concentração x tipo de estaca) utilizou-se 30 estacas, totalizado 270 estacas para a realização do experimento.

Os dados obtidos foram submetidos a análise de variância e os valores médios comparados entre si pelo teste de Tukey à $5 \%$ de probabilidade. Os gráficos foram gerados utilizando o software Sigma Plot 10.0.

\section{RESULTADOS E DISCUSSÃO}

Os efeitos das diferentes doses de AIB e dos tipos de estacas de Alfazema-do-Brasil estão apresentados na Tabela 1. Em propágulos sem aplicação de AIB, superioridade significativas foram observadas na taxa de sobrevivência, enraizamento e brotação nas estacas do tipo semi-lenhosa, em comparação com as classes herbácea e lenhosa. A aplicação de AIB nas doses de 1500 e 3000 ppm, praticamente, não resultou em estímulos significativos nos parâmetros avaliados, independente da classe de estaca, sendo observado efeito positivo apenas no aumento significativo do comprimento médio da maior 
raiz em estacas semi-lenhosas tratadas com AIB à 1500 ppm.

As maiores taxas de sobrevivência, enraizamento e brotação foram observadas em estacas semilenhosas sem adição de AIB. No entanto, de maneira geral, esta classe de estacas (semi-lenhosa) também apresentou melhor eficiência de propagação, em relação às demais, sob dose de 1500 e 3000 ppm de AIB.

Neste trabalho, as estacas herbáceas não sobreviveram, independente da concentração utilizado do fitoregulador. Este fator pode estar relacionado à fatores ambientais, e não corrobora com os resultados encontrados por Santos et al. (2009), que afirmam ter observado acima de 95\% de estacas vivas e com presença de enraizamento. Além disso, nas estacas herbáceas devido à proximidade com a região apical, maiores conteúdos de auxinas são encontrados e facilitaria o enraizamento (TAIZ et al., 2017). Sendo assim, o grande problema ocorrido pode estar relacionado às condições ambientais, período de duração do experimento e/ou problemas com a planta matriz.

Segundo Cunha et al. (2012), cada espécie de planta responde de maneira variável quanto a aplicação e o tipo de regulador. A adição de concentrações além do necessário, pode tornar-se inibitório à formação de raízes adventícias. Portanto, as concentrações de 1500 e 3000 ppm de AlB podem ter sido excessivas para estas condições experimentais, produzindo um efeito inibitório na formação de raízes adventícias, influenciando assim, nos outros parâmetros avaliados.

Analisando a Tabela 1, é possível observar que apenas o tipo de estaca desencadeou estímulos significativos sobre os parâmetros avaliados. A dose de AIB e a interação entre tipo de estacas e dose de AIB não resultou em mudanças significativas na produção de estacas.

Tabela 1: Efeito de diferentes doses de ácido indolbutírico (AIB) em três classes de estacas de Aloysia gratissima nas taxas de estacas vivas (EV), enraizadas (EE) e com brotações (EB), e no número de raízes por estaca (NRE) e comprimento médio da maior raiz (CMR).

\begin{tabular}{|c|c|c|c|c|c|c|}
\hline AIB (ppm) & Tipo de Estacas & EV & $\mathrm{EE}$ & EB & NRE & CMR \\
\hline & Herbácea & $0 \mathrm{~b}$ & $0 \mathrm{~b}$ & $0 \mathrm{~b}$ & $0 \mathrm{a}$ & $0 \mathrm{~b}$ \\
\hline \multirow[t]{3}{*}{0} & Semi-Lenhosa & $23,3 \mathrm{a}$ & $20,0 \mathrm{a}$ & $23,3 \mathrm{a}$ & $0,70 \mathrm{a}$ & $1,64 \mathrm{~b}$ \\
\hline & Lenhosa & $6,67 b$ & $\mathrm{Ob}$ & $6,67 \mathrm{~b}$ & $0 \mathrm{a}$ & $\mathrm{Ob}$ \\
\hline & Herbácea & $\mathrm{Ob}$ & $\mathrm{Ob}$ & $\mathrm{Ob}$ & $0 \mathrm{a}$ & $\mathrm{Ob}$ \\
\hline \multirow[t]{3}{*}{1500} & Semi-Lenhosa & $13,33 \mathrm{~b}$ & $10,0 \mathrm{~b}$ & $3,33 \mathrm{~b}$ & $0,80 \mathrm{a}$ & $4,40 \mathrm{a}$ \\
\hline & Lenhosa & $3,33 \mathrm{~b}$ & $\mathrm{Ob}$ & $3,33 \mathrm{~b}$ & $0 \mathrm{a}$ & $0 \mathrm{~b}$ \\
\hline & Herbácea & $\mathrm{Ob}$ & $\mathrm{Ob}$ & $\mathrm{Ob}$ & $0 \mathrm{a}$ & $\mathrm{Ob}$ \\
\hline \multirow[t]{2}{*}{3000} & Semi-Lenhosa & $6,67 b$ & $6,67 \mathrm{~b}$ & $6,67 b$ & $0,57 \mathrm{a}$ & $1,12 b$ \\
\hline & Lenhosa & $6,67 \mathrm{~b}$ & $3,33 b$ & $6,67 \mathrm{~b}$ & $0,033 a$ & $0,70 \mathrm{~b}$ \\
\hline CV (\%) & & 144,56 & 175,89 & 114,56 & 209,04 & 220,1 \\
\hline \multicolumn{7}{|c|}{ Valores de $F(P \leq 0,05)$} \\
\hline \multicolumn{2}{|c|}{ Tipo de Estacas (TE) } & $8,190^{*}$ & $6,727^{*}$ & $8,190^{*}$ & $5,889^{*}$ & $4,216^{*}$ \\
\hline \multicolumn{2}{|c|}{ Dose (AIB) } & $1,333^{\text {ns }}$ & $0,545^{\text {ns }}$ & $1,333^{\text {ns }}$ & $0,042^{\text {ns }}$ & $0,647^{\text {ns }}$ \\
\hline \multicolumn{2}{|l|}{ TE X AIB } & $1,238^{\mathrm{ns}}$ & $1000^{\text {ns }}$ & $1,238^{\text {ns }}$ & $0,068^{\text {ns }}$ & $1,001^{\text {ns }}$ \\
\hline \multicolumn{2}{|l|}{ Blocos } & $0,571^{\text {ns }}$ & $1,273^{\text {ns }}$ & $0,571^{\text {ns }}$ & $1,401^{\text {ns }}$ & $1,031^{\mathrm{ns}}$ \\
\hline
\end{tabular}

* Médias na coluna, seguidas da mesma letra, não diferem entre si, pelo teste de Tukey ( $p>0,05)$.

A Figura 1 representa graficamente o efeito do tipo de estaca sobre os parâmetros experimentais, sendo evidenciado que nenhuma estaca herbácea sobreviveu ao período de aclimatação. Em todas as características avaliadas, não foram observadas diferenças significativas entre estacas lenhosas e herbáceas, por mais que em quase todos os parâmetros foram observados valores maiores em estacas lenhosas.

As estacas semi-lenhosas apresentaram os melhores resultados para todos os parâmetros avaliados, 
apresentando superioridade significativa em comparação às estacas herbáceas em todas as características. Em relação às estacas lenhosas, a classe semi-lenhosa mostrou valores superiores, porém, apresentou aumento significativo apenas na taxa de estacas enraizadas e no número de raízes estaca-1.
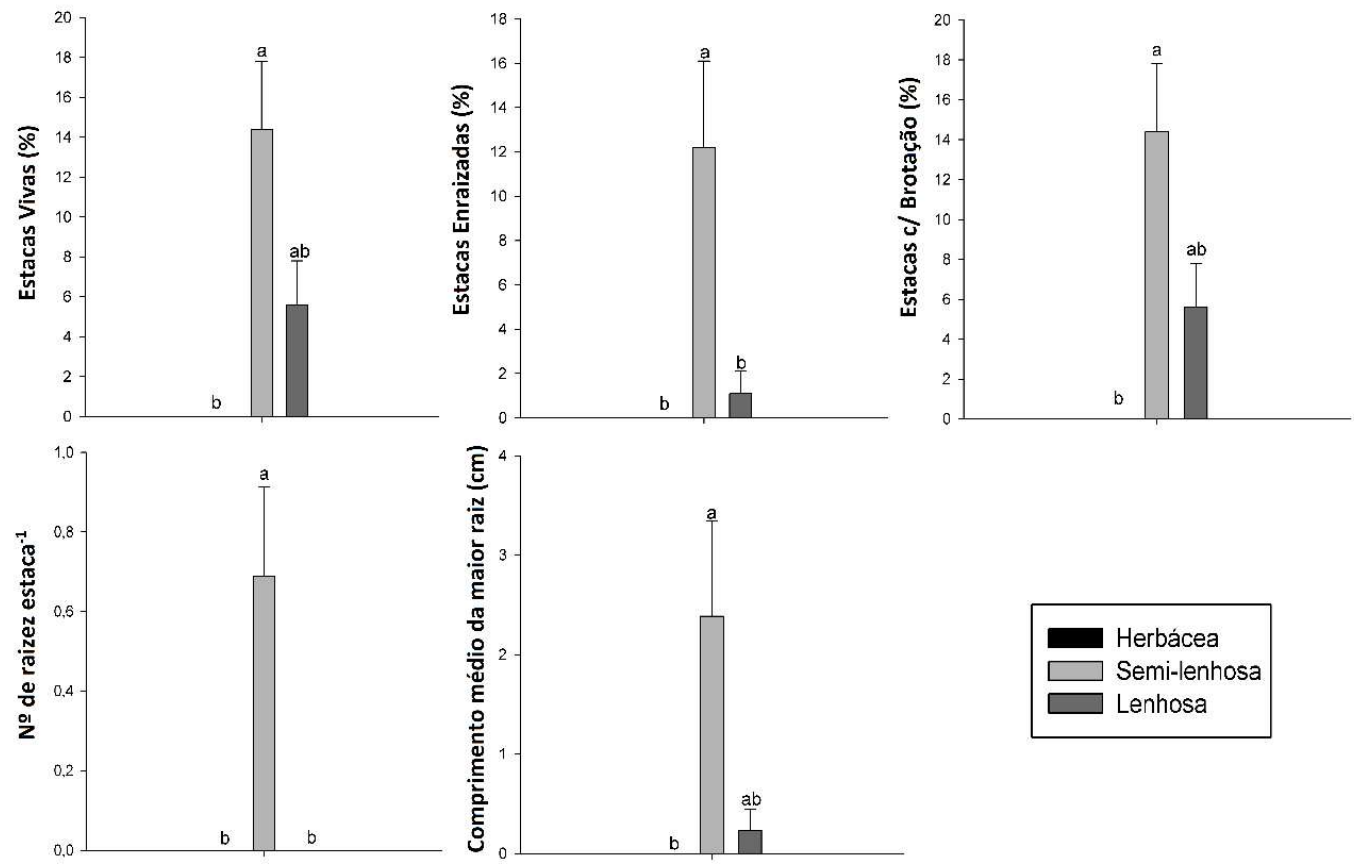

Figura 1: Efeito do tipo de estaca nas taxas de sobrevivências, enraizamento e brotação, e no número de raízes estacas e no comprimento médio da maior raiz.

Por mais que não tenha sido observado influência significativa das doses de AIB nos parâmetros avaliados, vale destacar os resultados encontrados (Figura 2). Analisando as taxas de sobrevivência, enraizamento e brotação das estacas, é possível observar que a aplicação de AIB nas doses de 1500 e 3000 ppm resultaram em menores valores, porém sem diferença significativa. Havendo ainda, valores menores de estacas vivas e com brotações na dose de 3000 ppm em comparação com a dose de 1500 ppm. Em relação ao número de raízes estaca-1 e ao comprimento médio radicular, foram observados maiores valores pela aplicação de AIB na dose de 1500 ppm, em relação as demais concentrações, sendo os únicos parâmetros em que foram observados algum estímulo, mesmo que não significativo, na produção das mudas.

Fazer o tratamento de estacas com indutores radiculares, como o AIB, pode aumentar a produção de mudas e melhorar a uniformidade do enraizamento (BOLIANI et al., 1998). Especificamente neste estudo, a adição de ácido indolbutírico não expressou resultados satisfatórios do ponto de vista agronômico. Entretanto, tais resultados necessitam de novos trabalhos para nortear a melhor forma de utilizar AIB. A baixa eficiência nos tratamentos com aplicação de indutor pode estar relacionada a outros fatores além da dose aplicada. De acordo com Pasqual et al. (2001), faz-se necessário haver um balanço hormonal endógeno para iniciar processo de iniciação radicular. Além disso, a concentração de indutor é variável conforme a espécie. 

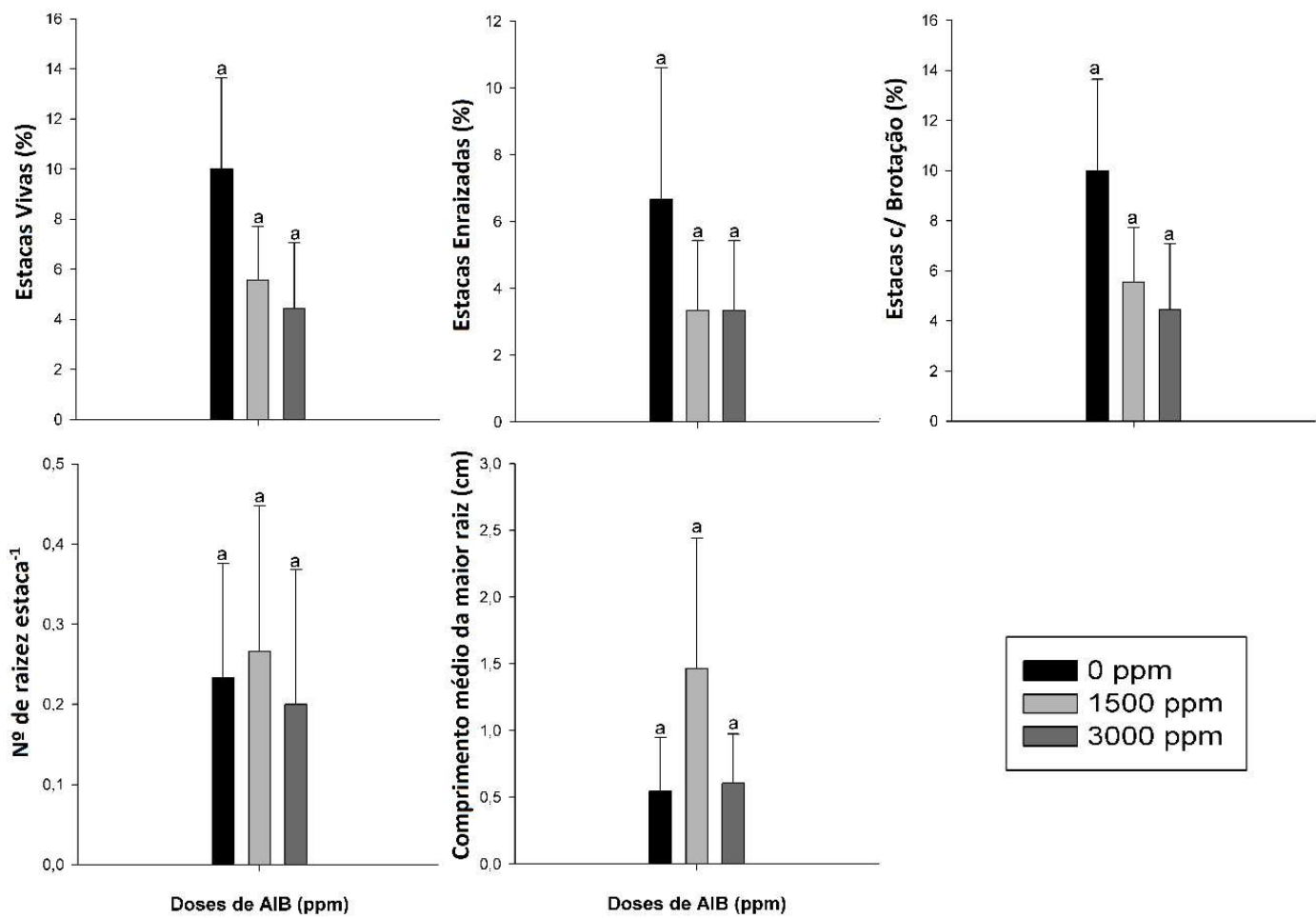

Figura 2: Efeito do tipo de estaca nas taxas de sobrevivências, enraizamento e brotação, e no número de raízes estacas $^{-1}$ e no comprimento médio da maior raiz.

\section{CONCLUSÕES}

A classe de estaca semi-lenhosa foi a que apresentou melhor eficiência na propagação de Alfazemado-Brasil. $\mathrm{O}$ tratamento controle $(0 \mathrm{ppm})$ apresentou resultados melhores para a maioria dos parâmetros analisados. A adição de AIB nos diferentes tipos de estacas não apresentou estímulos significativos, podendo estar relacionado à fatores ambientais, ao tempo de experimento para avaliação da produção das mudas, a qualidade da planta matriz/ou às concentrações utilizadas do fitorregulador não terem sidos as ideais para essa espécie.

Devem ser realizados mais trabalhos envolvendo a propagação por estaquia de Alfazema do Brasil, testando a aplicação de AIB em outras condições experimentais, para que assim seja realmente concluído que o uso deste fitorregulador não é adequado para a cultura.

\section{REFERÊNCIAS}

BOLIANI, A. C.; SAMPAIO, V. R.. Efeitos do estiolamento basal e do uso do ácido indolbutírico no enraizamento de estacas de nespereira(Eriobotrya japonica Lindley). Cultura Agronômica, v.7, n.1, p.51-63,1998.

CARDOSO, J. C. W.. Níveis de luz e homeopatia sobre caracteres morfofisiológicos e óleo essencbora ial e atividade fungitóxica do óleo essencial de Aloysia gratissima (Gilles \& Hook.) Tronc. Dissertação (Mestrado em Fitotecnia) - Universidade Federal de Lavras, Lavras, 2005.

CECHINEL FILHO, V.; YUNES, R. A.. Estratégias para obtenção de compostos farmacologicamente ativos a partir de plantas medicinais. Conceitos sobre modificação estrutural para otimização da atividade. Química Nova, v.21, n.1, p.99-105, 1998.
CUNHA, C. S. M.; MAIA, S. S. S.; COELHO, M. F. B.. Estaquia de Croton zehntneri Pax et Hoffm. com diferentes concentrações de ácido indolbutírico. Ciência Rural, v.42, n.4, p.621-626, 2012.

FACHINELLO, J. C.; HOFFMANN, A.; NACHTIGAL, J. C.; KERSTEN, E.; FORTES, G. R. L.. Propagação de plantas frutíferas de clima temperado. Pelotas: UFPel, 1995.

FERRIANI, A. P.; BORTOLINI, M. F.; ZUFFELLATO-RIBAS, K. C.; KOEHLER, H. S.. Propagação vegetativa de estaquia de azaléia arbórea (Rhododendron thomsonii HOOK. Semina: Ciências Agrárias, v.27, n.1, p.35-42, 2006.

KOEHN, F. E.; CARTER, G. T.. The evolving role of natural products in drug discovery. Nature Reviews Drug Discovery, v.4, n.3, p.206-220, 2005. 
PASQUAL, M.; CHALFUN, N. N. J.; RAMOS, J. D.; VALE, M. R.; SILVA, C. R.. Fruticultura comercial: propagação de plantas frutíferas. Lavras: UFLA, 2001

PEREIRA, G. H. A.; COUTINHO, F. S.; SILVA, R. A. C.; LOSS, A.. Desenvolvimento de estacas de alamanda amarela sob diferentes concentrações de ácido indolbutírico. Comunicata Scientiae, v.3, n.1, p.16-22, 2012.

RICCIARDI, G. A. L.; VAN BAREN, C. M.; LIRA, P. D. L.; RICCIARDI, A. I. A.; LORENZO, D.; DELLACASSA, E.; BANDONI, A. L.. Volatile constituents from aerial parts of Aloysia gratissima (Gillies \& Hook.) Tronc. var. gratissima growing in Corrientes, Argentina. Flavour Fragrance Journal, v.21, n.4, p.698-703, 2006.

SANTOS, F. M.; PINTO, J. E. B. P.; ALVARENGA, A. A.; OLIVEIRA, J. A.; OLIVEIRA, A. A.; OLIVEIRA, L. P.. Produção de mudas de Aloysia gratissima (Gillies \& Hook.) Tronc. por meio da propagação sexuada e assexuada. Rev. Bras. PI. Med., Botucatu, v.11, n.2, p.130-136, 2009.

SILVA, S. R. S.; BARBOSA, L. C.; DEMUNER, A. J.; ANDRADE, N. J.; PINHEIRO, A. L.. Análise de constituintes químicos e da atividade antimicrobiana do óleo essencial de Melaleuca alternifolia Cheel. Revista Brasileira de Plantas Medicinais, v.6, n.1, p.63-70, 2003.

TAIZ, L.; ZEIGER, E.; MOLLER, I. A.; MURPHY, A.. Fisiologia e desenvolvimento vegetal. 6 ed. Porto Alegre: Artmed, 2017.

YUNES, R. A.; PEDROSA, R. C.; CECHINEL FILHO, V.. Fármacos e fitoterápicos: a necessidade do desenvolvimento de fitoterápicos e fitofármacos no Brasil. Química Nova, v.24, n.1, p.147-152, 2001. 